\title{
Summarising Unreliable Data
}

\author{
Stephanie Inglis \\ Department of Computing Science \\ University of Aberdeen \\ r01si14@abdn.ac.uk
}

\begin{abstract}
Unreliable data is present in datasets, and is either ignored, acknowledged ad hoc, or undetected. This paper discusses data quality issues with a potential framework in mind to deal with them. Such a framework should be applied within data-to-text systems at the generation of text rather than being an afterthought. This paper also shows ways to express uncertainty through language and World Health Organisation (WHO) corpus studies, and an experiment which analyses how subjects approached summarising data with data quality issues. This work is still ongoing.
\end{abstract}

\section{Introduction}

Databases are used in multiple fields for various purposes. While gathering and using this data, issues arise regarding the quality of the data. These problems take multiple forms, and identifying them within a dataset can sometimes prove challenging or impossible (Daniel et al. 2008). Once identified, action needs to be taken. In a large database, amending all problem entries could be a costly task prone to human error, potentially creating more issues. Alternatively, it may not be possible to resolve the error. Either way, the user of the data must be informed of these errors if they are to use this data accurately.

Currently when companies use data to generate text, data quality issues are resolved ad hoc rather than during the generation phase. Instead, a framework should be created to deal with these issues at the point of generation, rather than amending the document if required.

First, we cover a discussion of related work followed by a corpus study of Ebola and Global Road Traffic reports provided by the WHO. An experiment is used to investigate further in Section 4. Finally we outline future steps.

\section{Related Work}

\subsection{Data Quality}

The quality of data impacts the amount of confidence we can have in our conclusions. By being aware of the issues within the data, we can begin to attempt resolution.

Daniel et al. (2008) discusses a system which aggregates reports in an attempt to improve the quality of reports hampered by poor quality data (see Figure 1). The data is acquired after summer from various sources such as hospitals, laboratories and emergency rooms. These reports are for the Italian Department of Health to predict the number drugs required over a winter period to treat flu, to prepare for outbreaks or to negotiate prices with manufacturers. Incorrect conclusions could result in overspending and the health department losing money, or not having enough drugs readily available for those who require them. The key problems are categorised as completeness, consistency and confidence issues. These are some issues likely to be missed by data cleaning tools.

Completeness covers missing data, which includes empty cells as well as entirely lost entries. This system ignores rows with the diagnosis field missing as these could result in false drug quantity estimations.

Consistency covers data that is not classified together but has the same meaning. The example entries in the paper show "influenza" and "flu" to be different diagnoses however they should be represented as the same. This can also occur through human error by mistypes which will also create a new, unwanted diagnosis e.g. "flu" being mistyped as "flyu". The precision of the diagnosis may result in additional entries such as including the type of flu of a patient. When ordering "influenza" drugs, underestimation may occur as "flu", "flyu" and "flu type A" may not be included in the count.

Thirdly, confidence shows how accurate data is. Rows may be fraudulent or erroneous leading to more incorrect estimations. Misinterpretations 


\begin{tabular}{|l|l|l|l|l|l|l|}
\hline ID & Diagnosis & Hospital & Province & $\ldots$ & Problem & Action \\
\hline 1 & Flu & San Raffaele & Milano & $\ldots$ & Refers to same therapy & Treat similarly \\
\cline { 1 - 4 } 2 & Influenza & Santa Clara & Trento & $\ldots$ & & \\
\cline { 1 - 3 } 3 & Flyu & San Raffaele & Milano & $\ldots$ & Mistyped & Interpret as "Flu" \\
\hline
\end{tabular}

Figure 1 - Examples of poor data quality and the action taken to deal with it.

of variable meanings also present issues. The example shown in the paper is "cost" of the drug differing for the same diagnosis. This could be interpreted by some as with tax added, while others omitted it.

While this is more of a consistency issue, its interpretation impacts the confidence of the data. A proposed solution is to replace an ambiguous variable with a more general variable, such as adjusting cost to become maximum cost.

\subsection{Vague Language}

Another aspect to investigate is the language used when conveying unreliable data, such as numerical data. When the author is writing a report and is unsure of the data, language becomes vague to allow for uncertainty. The reader will see these words and intuitively know that the author is not certain about their conclusion.

Van Deemter (2010) claims something to be vague if "it allows borderline cases", and subsequently defined categories of vague language. Adjectives themselves are vague as they allow borderline. Vague quantifiers such as "many", "most" or "few also allow borderline cases. For lack of specificity, the term is not vague by definition but a concrete value is not provided, such as "more than 5". Comparatives use degrees of adjectives such as " 30 is greater than 28 ". Finally, hedges express uncertainties by using words such as "appears", "suggests" or "may". This allows the author to make statements without committing to them as fact such as "numbers appear to be increasing".

This work looks at vagueness in the context of data quality issues as described above. For example, different vague language is used for missing data and inconsistent data.

\subsection{Real World Applications}

The use of vague language in low data quality situations is present in industry applications.

BT-Nurse is one application, generating handovers for nurses caring for pre-term neonates and

\footnotetext{
${ }^{1}$ World Health Organisation, Situation reports with epidemiological data: archive,
}

sick babies (Hunter et al, 2012). The handover is generated at the end of a shift so the next nurse on the ward knows the babies current conditions. High data quality is important as the health of the babies depend on it. An example of incomplete data is when a baby is intubated, but an accurate time is not recorded. To try to correct this, the ventilator mode data is checked. When an estimation is present in the text, phrases such as "around 19:45" and "by about 06:15" were used.

Sripada et al. (2014) discuss a system able to generate 50,000 high quality weather reports in less than two minutes. This system is used by the Met Office to generate reports for public use. As these are predictions, the further away the forecast, the greater the uncertainty in the data. Therefore the reports on day 3 have different language compared to those reports on day 1 . The paper shows this on practise where on day 3 the word "expected" is included, whereas this would be omitted if the forecast was for day 1 . The use of vague language helps to convey this uncertainty.

\section{WHO Ebola Reports}

Information can be communicated through various mediums, ranging from visual graphs to sentences. The $\mathrm{WHO}^{1}$ has followed the Ebola virus disease outbreak and provided detailed weekly reports and frequent updates on the situation. The reports used span from $29^{\text {th }}$ August 2014 to $4^{\text {th }}$ February 2015, containing 24 main weekly reports and 12 additional update reports. These reports contain a variety of tables, maps, graphs and sentences describing the number of suspected, probable and confirmed cases and deaths that have occurred in various countries as a result of the outbreak. The focus was primarily on the three most affected countries - Guinea, Liberia and Sierra Leone. An attempt was made to use the figures given in the tables to replicate sentences using the SimpleNLG (Gatt and Reiter, 2009) library. While doing so, the issues mentioned in section 2.1 arose.

http://www.who.int/csr/disease/ebola/situation-reports/archive/en/ . Last accessed 23rd June 2015. 


\begin{tabular}{|l|l|c|c|}
\hline Country & $\begin{array}{l}\text { Case } \\
\text { definition }\end{array}$ & Cases & Deaths \\
\hline Liberia & Confirmed & 950 & $*$ \\
\cline { 2 - 4 } & Probable & 1923 & $*$ \\
\cline { 2 - 4 } & Suspected & 1376 & $*$ \\
\cline { 2 - 4 } & All & 4249 & 2458 \\
\hline
\end{tabular}

"Data acquisition continues to be a challenge in Liberia. Evidence obtained from responders and laboratory staff in the country suggests that the situation in Liberia is getting worse"

Figure 2- A data and textual example taken from the Situation Report on the 15th October 2014. This shows an instance of missing data in deaths reported in Liberia.

\subsection{Data Quality}

Incompleteness was largely evident in Liberia's data (see Figure 2). No data was given from report 4 to 20 , covering almost 2 months. Throughout these reports, phrases such as "data acquisition continues to be a challenge" in report 14 can be found to describe Liberia's situation. Eventually, the data reached a quality so low that the same report quotes "problems with data gathering make it hard to draw any firm conclusions from recent data" whereas previously, WHO had at least speculated on trends in the data.

Inconsistencies exist between the numbers in the table and the text. Numbers were not mentioned explicitly in the text until around report 20. However, some vague statements appeared beforehand, such as "with over 200 new cases reported" on the 18th September. When numerical data was mentioned, it almost exclusively referred to the confirmed deaths. Data on Guinea was mostly inconsistent, with only 5 of the 26 reports being consistent between the tabular data and the textual data. One explanation is that reports were updated after publishing when late lab results were produced, but only for one layout.

Finally, there is evidence of lack of confidence. Data is incorrect in some situations, such as when the number of deaths exceeded the number of cases. This can be seen in report 12 on the $8^{\text {th }}$ October, occurring in both Liberia and Sierra Leone. Identifying data that is inaccurate will lower the confidence.

\footnotetext{
${ }^{2}$ World Health Organisation. (2009). Global status report on road safety 2009. Available:

http://www.who.int/violence_injury_prevention/road_safety_status/2009/en/. Last accessed 23rd June 2015.
}

\subsection{Vague Language}

The reports on Ebola have numerous examples of vague language being used.

Phrases such as "this is a genuine decline", "there may not yet be full agreement", and "based on the best information available" appeared frequently. The adjectives "genuine", "full" and "best" allow borderline cases, and so are vague. Therefore the phrases themselves are vague, and suggest this is more an opinion of the writer rather than fact. Vague quantifiers such as "many of the suspected cases", "there appears to be some evidence", and "very few confirmed cases were reported" also appeared often. Lack of specificity is rare but it does exist, for example "countries report that more than $80 \%$ ". The main comparative phrase in these reports is "it is too soon to say". Finally, examples of hedges include phrases that include the words "appears", "suggests" or "may". These words are used in the majority of the reports such as "appears to have stabilised", "which suggests that many of the suspected cases", and "which may lead to a revision of the numbers of cases and deaths".

Vague language was strongly used to describe the data in the Ebola reports. To investigate this further, an experiment was done using data from a different report, described in the next section.

\section{Pilot Study}

\subsection{Set Up}

To investigate human language in describing unreliable data, subjects were asked to summarise tables of data (see Figure 3). The experiment makes use of the Global Road Traffic reports for $2009^{2}$ and $2013^{3}$ provided by the WHO. A new domain was selected to observe differences between this corpus and the Ebola corpus, though none are identified yet (see Future Work).

Subjects were asked to assume the role of a news reporter on Twitter and report information to followers. Due to Twitter constraints, subjects were restricted to only 140 characters per country. This forced subjects to be concise and to prioritise the information given to them.

For 6 of the 183 possible countries, the number of deaths reported by the police, the number of estimated deaths, and a 95\% confidence interval

\footnotetext{
${ }^{3}$ World Health Organisation. (2013). Global status report on road safety 2013. Available: http://www.who.int/violence_injury_prevention/road_safety_status/2013/en/. Last accessed 4th June 2015 .
} 
were given. While the reported figures were provided by police data, the estimated deaths were produced by a model by the WHO, which applies negative binomial regression if the police data is less than $85 \%$ complete.

While the data was for real countries, they were renamed Country A to $\mathrm{F}$ to avoid bias. 22 subjects successfully completed the experiment, providing 132 tweets for analysis.

\section{Country C}

\begin{tabular}{|c|c|c|}
\hline $\begin{array}{l}2007 \\
\text { Reported } \\
\text { Deaths }\end{array}$ & $\begin{array}{l}2007 \\
\text { Estimated } \\
\text { Deaths }\end{array}$ & $\begin{array}{l}\text { 95\% Confidence } \\
\text { Interval }\end{array}$ \\
\hline 105,725 & 196,445 & $155,727-266,999$ \\
\hline $\begin{array}{l}2010 \\
\text { Reported } \\
\text { Deaths }\end{array}$ & $\begin{array}{l}2010 \\
\text { Estimated } \\
\text { Deaths }\end{array}$ & $\begin{array}{l}\text { 95\% Confidence } \\
\text { Interval }\end{array}$ \\
\hline 130,037 & 231,027 & Not reported \\
\hline
\end{tabular}

Subject 1

In 2010, there were 130,037 deaths reported of an estimated 231,027, up from 2007, when 105,725 deaths were reported out of 196,445.

Figure 3- An example of stimulus used in the experiment taken from the reports, and an example of a tweet given.

\subsection{Findings}

To evaluate the tweets, they were annotated by the first author to identify the different techniques subjects used to report information. These were:

- If the exact police or WHO numbers were used

- If a description of the numbers was used i.e. "around 300 deaths"

- If a trend in the data was mentioned

- If data quality was mentioned

- If opinions were given

No second annotator was present. The example in Figure 3 was annotated as police numbers, WHO numbers and a trend ("up from 2007").

As this is a pilot study, further study is needed to improve confidence in these findings. It was found that different subjects used different techniques $(\mathrm{p}<0.001$ for police numbers, WHO numbers, Descriptions and Opinions, $\mathrm{p}=0.007$ for trends using Pearson Chi-Squared). The only instance this did not apply to was data quality indicating subjects used this technique in a similar way. If data quality was mentioned by subjects, they were likely to add an opinion ( $\mathrm{p}=0.02$, Pearson Chi-Squared).

If data was incomplete, the quality of data was more significantly likely to be mentioned
( $<<0.001$, Pearson Chi-Squared), as well as more specifically that missing data was the quality issue ( $<<0.001$, Pearson Chi-Squared).

Unlike incomplete data, subjects were not significantly more likely to mention data quality if data was inconsistent $(p=0.157$, Pearson ChiSquared). However, when data was consistent, subjects were likely to acknowledge this $(p<0.001$, Pearson Chi-Squared). Subjects were also significantly likely to mention trends when the data was consistent ( $\mathrm{p}=0.01$, Pearson ChiSquared).

As there was no indication of how confident we could be in the data, there was no way to investigate if subjects' tweets correlated with the actual accuracy of the data. An observation however was that only one of the 16 mentions of confidence was positive. The remaining 15 were unconfident in the data.

Another notable result was trends and descriptions were correlated, and were used as a pairing in 53 of the 75 instances that either trends or descriptions appeared $(\mathrm{p}<0.001$, Pearson ChiSquared).

Of the 132 tweets, only one directly mentioned the confidence interval, so this element of the experiment was discarded.

\section{Future Work}

Analysis of the vague language used in the experiment tweets will be done, as well as language comparisons between the two WHO corpuses used in this paper. Further experiments will be conducted using mechanical turk. One will use 20 countries and 150 subjects while another will give 75 subjects only reported figures and a further 75 subjects only estimated figures to provide a base line for the first experiment. These will concentrate on the findings from the initial experiment. Another potential experiment will give subjects text and investigate if they can identify present data quality issues. The increase in results should allow a deeper analysis. After analysing the results and undertaking further research into more low quality datasets, a framework will be developed and generated text will be evaluated by human subjects. Improvements will be made to the framework based on the feedback of subjects. 


\section{References}

Daniel, F., Casati, F., Palpanas, T., Chayka, O. and Cappiello, C. (2008). Enabling Better Decisions Through Quality-Aware Reports In Business Intelligence Applications.

Gatt, A. and Reiter, E. (2009). SimpleNLG: A realisation engine for practical applications. Proceedings of ENLG-2009

Hunter, J., Freer, Y., Gatt, A., Reiter, E., Sripada, S. and Sykes, C. (2012). Automatic generation of natural language nursing shift summaries in neonatal intensive care: BT-Nurse. Artificial Intelligence in Medicine, 56(3), pp.157-172.

Sripada, S.G., Burnett, N., Turner, R., Mastin, J., and Evans, D. (2014). A Case Study: NLG meeting Weather Industry Demand for Quality and Quantity of Textual Weather Forecasts. INLG 2014.p1-5.

Van Deemter, K. (2010), Not Exactly: In Praise of Vagueness, Oxford University Press, Oxford, GBR. 